\title{
Facilitative priority effects drive parasite assembly under coinfection
}

Fletcher W. Halliday ${ }^{1 *}$, Rachel M. Penczykowski2 ${ }^{2}$, Benoit Barrès ${ }^{3,4}$, Jenalle L. Eck ${ }^{1}$, Elina Numminen $^{3}$, Anna-Liisa Laine ${ }^{1,3}$

${ }^{1}$ Department of Evolutionary Biology and Environmental Studies, University of Zurich, 8057, Zurich, $\mathrm{CH}$

${ }^{2}$ Department of Biology, Washington University in St. Louis, St. Louis, MO 63130 USA

${ }^{3}$ Organismal \& Evolutionary Biology Research Program, PO Box 65, FI-00014 University of Helsinki, Finland

${ }^{4}$ Université de Lyon, Anses, INRAE, USC CASPER, Lyon, France

*Co-first authors

Correspondence: Winterthurerstrasse 1908057 Zurich, fletcher.w.halliday@gmail.com, +41 44 635 4767; One Brookings Drive, St. Louis, MO 63130 USA, rpenczykowski@wustl.edu, +1 $314-935-8282$

Running head: Host-mediated interactions \& parasite assembly 


\section{Abstract}

Host individuals are often coinfected with diverse parasite assemblages, resulting in

3 complex interactions among parasites within hosts. Within hosts, priority effects occur when the

4 infection sequence alters the outcome of interactions among parasites. Yet, the role of host

5 immunity in this process remains poorly understood. We hypothesized that the host response to

6 first infection could generate priority effects among parasites, altering the assembly of later

7 arriving strains during epidemics. We tested this by infecting sentinel host genotypes of Plantago

8 lanceolata with strains of the fungal parasite, Podosphaera plantaginis, and measuring

9 susceptibility to subsequent infection during experimental and natural epidemics. In these

10 experiments, prior infection by one strain often increased susceptibility to other strains, and these

11 facilitative priority effects altered the structure of parasite assemblages, but this effect depended

12 on host genotype, host population, and parasite genotype. Thus, host genotype, spatial structure,

13 and priority effects among strains all independently altered parasite assembly. Then, using a fine-

14 scale survey and sampling of infections on wild hosts in several populations, we identified a

15 signal of facilitative priority effects, which altered parasite assembly during natural epidemics.

16 Together, these results provide evidence that within host priority effects by early arriving strains

17 can drive parasite assembly, with implications for how strain diversity is spatially and temporally

18 distributed during epidemics.

20 Keywords: disease ecology; priming; priority effects; biotic interactions; coinfection;

21 competition; immunity 


\section{Introduction}

23 The diversity of parasites - organisms that live in and on hosts, potentially causing

24 disease - may rival the diversity of all other organisms on earth ${ }^{1}$. In light of this diversity, it is

25 not surprising that host individuals are often infected with diverse parasite assemblages,

26 composed of multiple parasite species or multiple genetic variants ('strains') of the same species

27 2,3. Within hosts, interactions among coinfecting parasite strains can influence the dynamics of

28 drug resistance ${ }^{4}$, evolution of virulence ${ }^{5}$, and the magnitude of parasite epidemics ${ }^{6}$, with

29 implications for host health ${ }^{7}$. Thus, understanding how parasite strains interact in shared host

30 individuals may be important for predicting the spread of infectious diseases and ameliorating

31 their impact on host populations. Yet, measuring how interactions among parasites influence

32 natural epidemics is notoriously difficult, as this requires manipulating focal mechanisms of

33 interactions and documenting the structure of parasite assemblages as epidemics unfold ${ }^{2,8-13}$.

34 Using a parasitic fungus that infects a wild host plant, this study experimentally tests whether

35 parasite interactions that are mediated by the host response to initial infection alter the structure

36 of parasite assemblages within hosts under field conditions, and then leverages the results of

37 these experiments to explain how parasite strains assemble during a natural epidemic.

Multiple parasites that encounter the same host individual can interact during

39 simultaneous infections, known as coinfections ${ }^{12,14,15}$. One potential mechanism of interaction

40 among coinfecting parasites occurs when host immune responses to one parasite alter host

41 susceptibility to secondary infections of another parasite ${ }^{2,16-18}$. This mechanism can result in

42 either antagonism or facilitation among coinfecting parasites, and ultimately can alter parasite

43 epidemics ${ }^{12,19,20}$. The immune response to initial infection can suppress coinfection when

44 infection by one parasite activates immune signaling pathways that induce resistance to 
45 subsequent infections, in a process known by a variety of terms including immune priming, cross

46 protection, induced resistance, or cross-immunity ${ }^{21-25}$. Alternatively, an early arriving parasite

47 can facilitate coinfection by inactivating immune signaling pathways that protect hosts from

48 multiple parasites ${ }^{26,27}$. These effects can be temporary and spatially restricted within hosts ${ }^{28}$, or

49 systemic and persistent long after initial infection ${ }^{25}$. Both mechanisms of immune-mediated

50 interactions among parasites have been reported in plant and animal hosts $25,29,30$. These effects,

51 which have been predominantly tested in laboratory environments but see 18 , indicate that changing

52 host susceptibility may be one mechanism among many (e.g., altering susceptible host density,

53 pathogen transmission rate, or the duration of infection; ${ }^{31-33}$ ) through which the sequence and

54 timing of infections-can influence the structure of parasite assemblages.

55 The field of community ecology provides a framework for understanding how the

56 sequence of infection on host individuals might alter parasite assemblages as epidemics unfold

$57^{9,31,34-38}$. Specifically, interactions among parasites that are contingent on the sequence of past

58 events can be a consequence of priority effects within hosts. Within hosts, priority effects occur

59 when the per-capita strength of antagonism or facilitation among parasites is altered by their

60 sequence of arrival ${ }^{9,39}$. Priority effects, in turn can drive community assembly, thereby altering

61 the structure of parasite communities during natural epidemics ${ }^{32,36}$. Priority effects are expected

62 to occur most commonly when species exhibit high niche overlap and when early-arriving

63 species have large impacts on the availability of that niche ${ }^{35}$. A host comprises the entire niche

64 available to parasites during infection ${ }^{40,41}$, and thus coinfecting parasites often exhibit high niche

65 overlap ${ }^{42-44}$, particularly when parasite assemblages are comprised of coinfecting strains of the

66 same parasite species e.g., 4 . Although priority effects have been predominantly used to describe

67 community assembly in multi-species parasite assemblages ${ }^{\text {reviewed in } 31}$, these same principles may 
68 apply to parasite assemblages comprised of multiple strains ${ }^{3}$. By activating immune responses

69 that alter host susceptibility, early arriving strains can therefore determine the availability of the

70 shared host niche ${ }^{45}$; thus, the immune response to initial infection may drive priority effects

71 among parasite strains within hosts, thereby altering the structure of parasite assemblages within

72 hosts.

73 The degree to which the sequence and timing of infection influences parasite assemblages

74 might depend on the history of interactions between host and parasite populations ${ }^{12}$. This history

75 of interactions between host and parasite populations, which is typically measured through local

76 adaptation assays ${ }^{46,47}$, is commonly reflected by differences in the susceptibility of certain host

77 genotypes to certain parasite genotypes ${ }^{48}$. Interactions among sequentially arriving parasites

78 could also depend on host or parasite genotypes if a given host genotype is more or less sensitive

79 to infection by the first or second arriving parasite genotype ${ }^{49}$, or if the response triggered by the

80 first arriving parasite is genotype specific ${ }^{50,51}$. Thus, whether or not within-host priority effects

81 alter parasite epidemics might depend on complex interactions among host and parasite

82 genotypes. Consequently, it is essential to incorporate genotypic variation into studies of

83 sequential infection among parasite strains.

The host response to infection may alter parasite interactions and epidemics ${ }^{12,16,43}$,

85 which, in turn, can influence the structure of parasite assemblages as epidemics unfold ${ }^{52}$.

86 However, variation in host genotype, the sequence of parasite arrival, and environmental

87 conditions, all strongly influence the probability of successful arrival, establishment, and

88 transmission from a host during epidemics ${ }^{48}$. Consequently, directly linking parasite interactions

89 with the structure of parasite assemblages as epidemics unfold requires studies that can

90 simultaneously manipulate prior parasite exposure and measure the consequences for parasites in 
91 natural populations ${ }^{11,13,53}$. Isolating the host response to infection as a driver of this process

92 requires studies that can additionally distinguish host-mediated interactions among parasites

93 from other types of interactions, like resource competition. Understanding how the host response

94 to infection alters the structure of parasite assemblages therefore remains a major research gap.

95 This study addresses this research gap experimentally by first infecting host plants with parasitic

96 fungi, physically restricting those parasites from interacting directly within hosts, and then

97 testing whether the host response to initial infection alters the structure of parasite assemblages.

98 We then leverage the experimental results to explain how parasite strains assemble within hosts

99 during a natural epidemic. We find that parasites exhibit facilitative priority effects driven by the 100 host response to initial infection, and that these facilitative priority effects can alter the structure

101 of parasite assemblages during a natural epidemic. These results indicate that the sequence of

102 infection can determine the probability of coinfection, altering the trajectory of parasite

103 assembly, and leading to pronounced differences in the structure of parasite assemblages among 104 hosts.

105

106 Results \& Discussion

107 In order to examine the role of priority effects among parasites that are mediated by the

108 host (i.e., plant) in response to prior infection and the influence of priority effects on the structure

109 of parasite assemblages, we carried out two field experiments, referred to as the "common

110 garden experiment" and the "natural epidemic experiment", and a fine-scale survey and sampling

111 of infections in the wild, referred to as the "wild host survey", using the focal host Plantago

112 lanceolata, and the obligate parasite Podosphaera plantaginis (Fig 1). 
Can host responses to prior infection drive parasite assembly via priority effects?

We carried out the common garden experiment in the field at the Lammi Biological

116 Station to test whether parasite strains exhibit priority effects that are mediated by the host

117 response to initial infection (Fig. 1). Both the host and parasite species naturally occur in this

118 location. In the common garden experiment, four host genotypes were either inoculated or mock-

119 inoculated with one of four parasite strains, which were sealed inside mesh pollination bags to

120 prevent direct strain interactions, and then exposed to all four priming strains for four days. To

121 be consistent with previously published literature ${ }^{54-57}$, we refer to the experimental treatment as

122 the "priming treatment" and the experimentally inoculated strains as "priming strains" (Fig. 1).

One challenge of predicting how within-host interactions will alter infection outcomes

124 during epidemics is the difficulty of isolating host-mediated interactions from other interactions

125 among parasites, such as resource or interference competition ${ }^{2,13,58}$. We overcame this limitation

126 experimentally by leveraging the modular growth form of plant hosts. Specifically, for foliar

127 parasites in plant hosts, resource and interference competition are expected to be strongest within

128 individual host leaves ${ }^{12,36,59}$. Because powdery mildews only feed within individual host leaves

$129{ }^{60}$ and the priming strain was restricted from spreading beyond the inoculated host leaf onto the

130 rest of the host plant, any response to experimental inoculation can be interpreted as an effect

131 that is mediated by the host response to initial infection. Thus, the inoculation treatment was

132 intended to test whether initial infection by one parasite could "prime" the host to respond

133 differently upon subsequent exposure, generating priority effects mediated by the host.

We tested whether the priming treatment altered the probability of a host becoming

135 infected in the common garden using a logistic mixed model. As predicted, hosts that were

136 experimentally inoculated were more likely to become subsequently infected during the 
137 experimental epidemic $(p=0.0088$; Fig. 2a; Supplementary Table 1a). This effect was 138 qualitatively similar using the (logit-transformed) proportion of leaves infected as a response 139 measure representing infection severity $(\mathrm{p}=0.019$; Supplementary Table 1b). Although host 140 susceptibility to infection and the severity of infection were positively influenced by the priming 141 treatment, this effect disappeared when we evaluated infection severity among infected hosts 142 only ( $\mathrm{p}=0.82$; Supplementary Table $1 \mathrm{c})$, suggesting that priority effects may act qualitatively 143 (e.g., by altering susceptibility to infection) rather than quantitatively (e.g., by altering infection 144 severity). This result therefore suggests that increased susceptibility to infection following early 145 exposure to a pathogen strain can influence subsequent infection outcomes in the field. We next tested whether the facilitative effect of early exposure on susceptibility to

147 infection during the experimental epidemic differed among host genotypes and priming strains.

148 Consistent with theory grounded in the history of interactions between host and parasite 149 populations ${ }^{\text {e.g., }} 12$, the facilitative effect of early infection depended on the priming strain $(\mathrm{p}=$ $1500.050)$ and host plant genotype $(p=0.024)$, though there was no interaction between host plant 151 genotype and the priming treatment ( $\mathrm{p}=0.86$; Supplementary Table 2a, Fig. 2c). We therefore 152 dropped the non-significant interaction, resulting in a reduced model, and estimated the 153 coefficients from the reduced model. Consistent with facilitative priority effects, the priming 154 strains G46 and O49 significantly increased the probability of infection under field conditions ( $p$ $155=0.039$ and $\mathrm{p}=0.016$, respectively), while strain O10 marginally significantly increased the 156 probability of infection $(\mathrm{p}=0.051)$ and strain $\mathrm{O} 15 \mathrm{did}$ not $(\mathrm{p}=0.62)$. This result suggests that 157 only some early arrivers strongly influenced the availability of the niche for later arrivers. These 158 results were qualitatively similar using the proportion of leaves infected as a metric of infection 159 severity (Supplementary Table 2b). Although the effect of the priming treatment on host 
susceptibility to infection and the severity of infection were influenced by host and parasite genotype, this effect disappeared when we evaluated infection severity among infected hosts

162 only (priming strain $p=0.95$; host plant genotype $p=0.61$; Supplementary Table $2 c$ ), lending

163 further support to the idea that priority effects act qualitatively rather than quantitatively in this 164 system.

165 Together, these results suggest that the host response to initial infection depended on 166 which parasite strain arrived first. However, for priority effects to alter the structure of parasite 167 assemblages, later arriving strains must also be sensitive to the plant response to initial infection 168 35. To explore this mechanism of within-host interactions, we next genotyped infections on each 169 individual host following exposure to all four strains under natural conditions and then tested for 170 interactions among the priming treatment, plant genotype, and whether or not the later arriving 171 strain was the same as the early arriving strain. For priority effects to occur, facilitative effects

172 should occur among different strains. In other words, a priority effect could only occur if the 173 early arriving strain facilitated other later arriving strains. Across both treatments, infection by a 174 non-priming strain was about 1.4 times more likely than infection by the priming strain assigned 175 to the tray ( $\mathrm{p}<0.001$; Fig $2 \mathrm{~b}$ ), which was expected, since each host was exposed to one priming 176 strain (assigned to the tray) and three non-priming strains simultaneously, regardless of whether 177 that host was inoculated (i.e., primed) or mock-inoculated (control). Consistent with the 178 hypothesis that parasite strains can exhibit within-host priority effects, the effect of early 179 infection on the probability of subsequent infection was qualitatively similar between secondary 180 infections caused by the priming strain $(\mathrm{p}=0.004)$ and secondary infections caused by a 181 different strain from the priming strain $(p=0.033)$. In other words, there was a significant main 182 effect of the priming treatment $(p=0.003)$, but no interaction between the priming treatment and 
183 whether or not the host became infected with a strain other than the priming strain $(\mathrm{p}=0.24$;

184 Supplementary Table 3).

Finally, we tested whether within-host priority effects altered the structure of parasite assemblages using a multivariate generalized linear model ${ }^{61,62}$. Multivariate generalized linear

187 models, which have been commonly used in studies of experimental community assembly e.g., 63-

$188{ }^{66}$, are similar to distance-based analyses of community structure, but with higher power, reduced 189 bias, and the ability to test for statistical interactions ${ }^{61}$. As expected, different parasite 190 assemblages formed on hosts that received different priming treatments (LRT $=37 ; p=0.040$; 191 Supplementary Table 4; Fig. 2d), though there were no significant differences among different 192 host genotypes $(\mathrm{LRT}=34.94 ; \mathrm{p}=0.085)$, and the effect of priming treatments on the structure of 193 parasite assemblages did not interact with host genotype (LRT $=57 ; \mathrm{p}=0.077)$. Thus, priority 194 effects among strains altered parasite assembly, and the trajectory of assembly depended on the 195 identity of the early arriving strain, but not the genotype of the host.

Can host-mediated priority effects among parasites influence parasite assembly during a natural 198 epidemic?

The common garden experiment tested whether hosts could mediate priority effects

200 among parasites, and whether such priority effects could influence parasite assembly during an 201 experimental epidemic. We next carried out the natural epidemic experiment (Fig 1) to test 202 whether host-mediated priority effects could be generalized to predict the outcome of natural 203 epidemics by embedding sentinel hosts that were either primed or mock-inoculated into an 204 ongoing epidemic in three wild host populations in the Åland archipelago. In addition to 205 manipulating infection sequence (primed vs mock-inoculated), this experiment also manipulated 
206 the timing of prior infection by priming hosts either four or eight days prior to exposing hosts to 207 the natural epidemic.

We first tested whether the host response to prior infection could generate priority effects using a logistic mixed model. Consistent with expectations from the common garden experiment, the priming treatment significantly influenced the probability of a host becoming infected during

211 the natural epidemic experiment ( $\mathrm{p}<0.001$; Supplementary Table 5). However, in contrast with

212 the common garden experiment, there was no significant effect of host genotype $(p=0.34)$.

213 Consistent with expectations grounded in previous studies of this system ${ }^{67}$, the probability of 214 infection differed among populations $(\mathrm{p}=0.002)$. But, in contrast with expectations grounded in 215 laboratory studies of plant immunity ${ }^{68}$ and ecological theory ${ }^{34}$, there was no difference in the 216 magnitude of the priming effects between hosts that were primed eight days prior to 217 experimental placement in the field and hosts that were primed four days prior to placement in 218 the field (Fig 3a).

In contrast to the results testing the probability of a host becoming infected during the 220 natural epidemic experiment, there was a significant three-way interaction in the model of 221 infection severity, using the (logit-transformed) proportion of leaves infected as a response 222 variable representing infection severity. Thus, the model of infection severity suggests that 223 priority effects occurred, that these priority effects depended on infection sequence and timing,

224 but only among certain host genotypes in certain populations (e.g., in the P1 treatment, 225 subsequent infection was facilitated most strongly by Genotype 511_14 in Population 294, by 226 Genotype 4_14 in Population 490, and by Genotype 9031_19 in Population 9066; Extended Data 227 1a). The reduced model of infection severity among infected hosts also included significant two228 way interactions between population and host genotype $(p=0.033)$, and between host genotype 
229 and experimental treatment $(\mathrm{p}=0.019$; Extended Data $1 \mathrm{~b})$. Several potential mechanisms could 230 generate population or genotype-level specificity in priming effects. For example, genotype231 specific host susceptibility ${ }^{69}$ or gene expression ${ }^{70}$ could alter priming effects by determining 232 susceptibility or immune responses to early-arriving parasites. The structure of the host 233 microbiome also often varies among host genotypes ${ }^{71,72}$, generating genotype-specific responses 234 to parasite infection ${ }^{73}$, suggesting that higher-order interactions among parasites and the resident 235 microbiome could also determine genotype-specific priming effects.

236 Our result that host-mediated interactions among parasites almost universally favored 237 coinfection is in contrast to prior studies suggesting that priming can commonly reduce the 238 probability of coinfection through cross resistance $22,25,74$, potentially raising concerns that these 239 results might be system specific; however, a prior study in this wild plant pathosystem suggests a 240 different explanation for these contrasting results. Specifically, using different Plantago

241 lanceolata hosts and Podosphaera plantaginis genotypes, Laine ${ }^{54}$ found that priming reduced 242 spore production in the lab, but increased infection severity in the field. This apparent tradeoff 243 between spore load production in the lab but increased productivity in the field, could also result 244 from genotype-specific immunity or selection for the microbiome. For example, parasite 245 passaging experiments often detect host-mediated genotype-specific selection for particular 246 spores ${ }^{75}$, suggesting that there might be tradeoffs between spore quality and quantity in the field.

247 Additionally, studies conducted in the laboratory might overlook important microbiome-

248 mediated interactions, especially if there is host genotype-mediated selection in microbiome that 249 alters host-parasite interactions ${ }^{71,73}$.

250 Just as immune-mediated cross resistance is a commonly hypothesized mechanism of 251 antagonistic priority effects among parasites, immune-based mechanisms could also explain why 
252 host-mediated priority effects were commonly facilitative in this experiment. For example,

253 facilitative priority effects may have resulted from immune suppression by early arriving strains,

254 resulting in systemic induced susceptibility to later arriving strains. Such a phenomenon was

255 recently demonstrated for a fungal pathogen of wheat, which suppressed production of

256 metabolites involved in immunity, altering the leaf microbiome and facilitating later infections

$257{ }^{76}$. Bacterial pathogens have also been shown to induce systemic susceptibility in herbaceous

258 plants, through a mechanism involving a pathogen-derived functional and structural mimic of

259 Jasmonic acid, which antagonizes the Salicylic-acid signaling pathway involved in systemic

260 acquired resistance responses ${ }^{77}$. Future studies could test these immune-mediated mechanisms

261 by combining experimental manipulations of immune signaling hormones ${ }^{\text {e.g., }} 18$ with

262 experimental inoculations in the field.

263 Finally, we tested whether priority effects among parasite strains could lead to variation

264 in the structure of parasite assemblages among hosts during a natural epidemic using a

265 multivariate generalised linear model. This model did not include any significant interactions.

266 However, as expected, there were different parasite assemblages on hosts that received different

267 priming treatments (LRT $=111 ; \mathrm{p}<0.001)$, among different populations (LRT $=90 ; \mathrm{p}<0.001$ ),

268 and among different host genotypes (LRT $=44 ; \mathrm{p}=0.048$; Supplementary Table 6; Fig 3b;

269 Extended Data 2). Thus, host genotype, spatial structure, and priority effects among strains all

270 independently altered parasite assembly in the natural epidemic experiment.

271

272 Can a signal of host-mediated facilitative priority effects among parasites be detected in natural 273 populations? 
Our experimental results showed consistent host-mediated facilitative priority effects

275

276

277

278

279

280

281

282

283

284

285

286

287

288

289

290

291

292

293

294

295

296

among parasite strains. However, in addition to host-mediated interactions, parasites can also interact via resource or interference competition during natural epidemics ${ }^{2}$. In theory, the hostmediated interactions can be either antagonistic or facilitative, whereas resource and interference competition are generally expected to reduce the risk of coinfection ${ }^{18,78,79}$. Thus, although both experiments suggested that the host response to prior infection can facilitate subsequent infection via within-host priority effects, the degree to which this process plays out to influence parasite assemblages during natural epidemics remains unclear.

We tested whether host-mediated priority effects are sufficiently important to influence the structure of parasite assemblages in nature by analyzing the results of a longitudinal survey of infection on wild host individuals during a natural epidemic (i.e., the wild host survey; Fig 1). The wild host survey was carried out in the Åland archipelago, and included 105 host individuals from 13 populations, sampled biweekly for infection starting on 7 July, 2014. Once a host became infected, it entered the dataset as a focal host. To determine infection sequence among hosts, we sampled lesions and genotyped infections twice on each focal host: first when more than one leaf on a focal host was infected, and then again at the end of the season. These two genotyping sessions provide data on the sequence and timing of infection among hosts, while biweekly surveys of whole host populations provide information on parasite phenology. We hypothesized that if the host response to prior infection was sufficiently strong to alter parasite community assembly, then we would observe a signal of facilitation among cooccurring parasite strains during a natural epidemic. To test whether parasite strains exhibit priority effects within hosts, we first fit a series of cox proportional hazards models following Halliday et al ${ }^{18,36}$. These models test whether the time until infection by each parasite strain was 
297 influenced by whether or not a host had been previously infected by another strain. In total this 298 analysis tested for 286 potential pairwise interactions among parasite strains. Overall, early 299 infection tended to facilitate subsequent infection by other strains more commonly than 300 preventing subsequent infection by other strains ( $<<0.001$; Fig $4 a)$, consistent with priority 301 effects being mediated by the host response to prior infection. However, only three out of 286 302 potential pairwise interactions among parasite strains resulted in a significant priority effect as 303 defined by Halliday et al ${ }^{18,36}$, suggesting that, individually, parasite strains were not exhibiting 304 measurable priority effects within hosts. Nevertheless, these results support the hypothesis that 305 facilitative interactions among parasite strains, mediated by the host response to prior infection, 306 would result in a signal of facilitation among co-occurring parasite strains.

307 Finally, to test whether parasite phenology among strains altered parasite assembly within 308 hosts, we fit a multivariate generalised linear model. We hypothesized, in accordance with 309 ecological theory e.g., 35 and our experimental results, that strains that emerged later in the 310 growing season (i.e., strains with later phenology) would be more sensitive to facilitative priority 311 effects, and that strains that emerged earlier in the growing season (i.e., strains with early 312 phenology) would more strongly influence parasite assembly. Consistent with this hypothesis, 313 the structure of parasite assemblages differed significantly among hosts with differing phenology 314 of the early-arriving strains (LRT $=15.23 ; \mathrm{p}=0.002$; Fig $4 \mathrm{~b}$; Supplementary Table 7), even after 315 accounting for survey date $(\mathrm{LRT}=238 ; \mathrm{p}=0.001)$, and host different population (LRT $=189 ; \mathrm{p}$ $316=0.001)$. Together, these results suggest that strains with early phenology might more commonly 317 facilitate subsequent infections, and that strains with later phenology might be more sensitive to 318 facilitation. In natural populations, strains of $P$. plantaginis vary in their ability to overwinter, 319 and in the timing of life-history events leading to transmission ${ }^{80,81}$. Early-phenology strains 
320 likely represent strains that successfully overwintered within the local population, and that are

321 quick to complete their life-cycle. While early-phenology, fast pace-of-life strains also tend to be

322 the strains with high overwintering potential, laboratory studies indicate that this life-history

323 strategy renders strains more sensitive to a mycoparasite, thus potentially imposing a cost that

324 helps maintain variation in local populations of $P$. plantaginis ${ }^{82}$. Our results suggest a potential

325 second mechanism helping to maintain variation in local populations: infection of hosts by early

326 phenology strains might facilitate subsequent infection by slower pace-of-life strains with later

327 phenology. Jointly these results therefore support the idea that the life-history variation typical of

328 many pathogens ${ }^{83}$ can contribute to the strain assembly process within populations, with far-

329 reaching implications for epidemiological dynamics.

330

331

\section{Conclusions}

333

334

335

336

337

338

339

340

341

342

343

The sequence and timing of infection can strongly influence parasite interactions and epidemics $18,31,32,36$, yet the degree to which this process is driven by the host response to infection versus other mechanisms of interaction among parasites remains poorly understood. This study leveraged a model wild-plant pathosystem to fill this gap ${ }^{84}$. Specifically, our study revealed three key findings: (1) by manipulating infection sequence during a common garden experiment, we showed that host-mediated interactions among parasites almost universally favored coinfection; (2) by manipulating infection sequence during a natural epidemic, we showed that this process could alter how parasite strains assemble; and (3) by tracking wild host individuals during the course of a different natural epidemic, we identified a signal of hostmediated facilitation among parasite strains that could be linked to the structure of parasite assemblages. Our results therefore provide comprehensive evidence that parasite interactions, 
344 mediated by the host response to initial infection, can facilitate subsequent infection by different 345 parasite strains, altering the trajectory of parasite assembly during natural epidemics.

347 parasites, our experimental approach overcomes an additional key limitation of past

348 observational studies: the need to rely on counterfactual reasoning. Specifically, priority effects,

349 by definition require researchers to ask "what if" questions (e.g., what would the outcome look

350 like if the early arriving species had arrived later?). These counterfactuals can be difficult to

351 measure in observational studies, particularly when the sequence of infection is determined by

352 abiotic conditions ${ }^{36}$. Thus, linking infection sequence and within-host priority effects during

353 natural epidemics is notoriously challenging ${ }^{13,85}$. Our experimental data showed clear evidence

354 of host-mediated facilitation among parasites. Pairing this experimental approach with a fine-

355 scale survey and sampling of infections in the wild during a natural epidemic thus allowed us to

356 interpret a signal of host-mediated facilitation that would be uninterpretable from observational

357 data alone. Our results suggest that, by differentially determining the plant response to infection 358 and experiencing differential sensitivity to that response, prior infection can strongly alter the 359 structure of parasite assemblages during epidemics.

Priority effects among strains favored coinfection and altered parasite assembly, and the 361 trajectory of assembly depended on the genotype of the early arriving strain. This result supports 362 the idea that species interactions - in this case host-parasite and parasite-parasite interactions 363 can depend on intraspecific variation in characteristics of organisms ${ }^{86-88}$. Intraspecific diversity 364 is ubiquitous in host and parasite populations, and has prompted considerable research into local 365 adaptation among hosts and parasites ${ }^{46}$, parasite aggregation ${ }^{89}$, and disease emergence ${ }^{90}$. Yet, 366 how this diversity impacts parasite assembly is not known. Importantly, our results suggest that 
367 by influencing host susceptibility, intraspecific variation can determine how strain diversity is 368 spatially and temporally distributed during epidemics.

369 In our experimental manipulation of a natural epidemic, we found evidence that infection

370 sequence, but not infection timing altered future host susceptibility. We also found strong

371 evidence that host genotype and spatial structure altered the structure of parasite assemblages

372 within hosts, but these effects did not alter the direction of priority effects and only altered the

373 magnitude of priority effects in models of infection severity. We expected priming effects to

374 exhibit genotype specificity, because host susceptibility, infection severity, and even gene

375 expression among infected hosts are consistently genotype-specific in this system ${ }^{69,70}$. However,

376 in this study, host genotype only weakly modulated priority effects, highlighting the need for

377 future studies to better elucidate the mechanisms of host-mediated interactions among parasites.

378 Nevertheless, we conclude that differences among host genotypes and among populations may

379 play a large role in the assembly of parasite strains in natural populations, but these effects are 380 largely independent of the robust effect of facilitation by sequentially arriving parasite strains in 381 this system.

382

383 Methods

384 Study system

385 The host plant, Plantago lanceolata L. (ribwort plantain), is a perennial rosette-forming

386 herb that reproduces either sexually, as an obligate outcrosser with wind-dispersed pollen, or via 387 vegetative propagation of side-rosettes ${ }^{91,92}$. This species has a cosmopolitan distribution, and 388 grows in fragmented populations on dry meadows and pastures in the Åland archipelago (SW 389 Finland) ${ }^{93}$. Plantago lanceolata is host to the obligate parasite Podosphaera plantaginis 
390 (Castagne; U. Braun and S. Takamatsu), a powdery mildew fungus in the order Erysiphales 391 within the Ascomycota. The fungus grows on the leaf surface and extracts plant nutrients via 392 haustoria that enter the epidermis ${ }^{60}$. On the leaf surface, mycelia produce chains of asexual, 393 wind-dispersed transmission spores (conidia). The parasite reproduces clonally throughout the 394 summer growing season, and then produces resting structures (chasmothecia) via haploid selfing 395 or outcrossing ${ }^{94}$ which enable the parasite to survive when the host plant has died back to 396 rootstock in winter. Within the chasmothecia, haploid ascospores develop, which re-initiate 397 epidemics in spring 95.

398 The metapopulation dynamics of this host-parasite interaction in ca. 4000 populations in 399 Åland have been studied since the year $2001^{96,97}$. Powdery mildew infection combined with 400 stressful environmental conditions can cause high mortality of Pl. lanceolata ${ }^{98}$, and infection 401 reduces host population growth rates ${ }^{98,99}$.

402 Successful infection is the outcome of a high degree of specificity where a given $\mathrm{Pl}$. 403 lanceolata genotype can be susceptible to some Po. plantaginis strains while able to block 404 infection by others ${ }^{98,100}$. Hosts that are qualitatively susceptible to a given parasite strain may 405 still vary in their ability to mitigate its sporulation once infected (i.e., quantitative resistance). 406 Evidence for diversity within and among host populations comes from laboratory inoculation 407 experiments showing variation in resistance to a given set of parasite strains among clonal plant 408 lines under controlled conditions ${ }^{98,100}$. The Po. plantaginis populations in Åland are also diverse, 409 comprised of genetically and phenotypically distinct parasite strains ${ }^{101}$, with a high proportion of 410 coinfection by different multilocus genotypes (MLGs) ${ }^{6}$.

\section{Study design}


414 infections in the wild (Fig. 1). In the first experiment, which we refer to as the "common garden

415 experiment", we tested whether priority effects, mediated by the host plant response to prior

416 infection, were specific to parasite strain. This study, therefore measured parasite interactions at

417 the scale of the whole host plant, rather than at the scale of individual infected leaves ${ }^{18, \text { e.g., }}{ }^{36}$. We

418 addressed this question by first inoculating or mock-inoculating hosts of four different genotypes

419 with one of four parasite strains and then later exposing the same hosts to all four strains

420 simultaneously in a common garden environment. In the second experiment, which we refer to as

421 the "natural epidemic experiment", we tested whether host-plant mediated priority effects among

422 parasite strains can influence parasite assembly during a natural epidemic. We addressed this

423 question by first inoculating or mock-inoculating hosts with one of three different parasite

424 strains, then embedding those hosts into one of three wild host populations in order to expose the

425 hosts to a natural epidemic, and then compared the effect of these treatments on the structure of

426 the resulting parasite assemblage on each sentinel host plant as well as wild plants from the same

427 populations. These experiments revealed strong facilitative priority effects among parasite

428 strains, and indicated that these facilitative priority effects can alter parasite assembly during

429 natural epidemics. Finally, we analyzed the results of an observational study, which we refer to

430 as the "wild host survey," to test whether a signal of host-plant mediated facilitative priority

431 effects is detectable among parasite strains in natural populations. We addressed this question by

432 tracking infections on wild host plants over the course of a natural epidemic.

434 Experimental plant genotypes. To test whether the outcome of sequential infections

435 varied across different host plant genetic backgrounds, we used a set of four host plant maternal 
436 lines in both experiments (Supplementary Table 8). Each maternal line came from a single seed

437 head from a different mother plant in the Åland archipelago. Seeds were sown in 9 x $9 \mathrm{~cm}$ flower

438 pots in a mixture of $30 \%$ sand, and $70 \%$ potting soil. Plants were maintained in the greenhouse at

$439+20^{\circ} \mathrm{C}$ until transport to the common garden location, where they were acclimated to outside

440 conditions for at least two weeks before the start of the experiments.

441

442

Experimental parasite strains. The parasite strains used for inoculating plants in both

443 experiments were collected from field populations in the Åland archipelago at the end of

444 epidemics in 2014 (Supplementary Table 9). We collected and purified the powdery mildew

445 isolates as follows. Infected leaves were detached using forceps and placed into $9 \mathrm{~cm}$ Petri dishes

446 containing moist filter paper. Between every sampled leaf, the forceps were sanitized with DNA-

447 Away (Molecular Bio Products) to avoid cross-contamination. To ensure that each parasite

448 isolate was a single strain (multi-locus genotype, MLG), we purified the isolates through three

449 successive single-colony transfers of spores onto detached, greenhouse-grown leaves ${ }^{102}$.

450 Inoculated leaves were maintained on moist filter paper in Petri dishes in a growth chamber

451 under standard conditions of $21^{\circ} \mathrm{C}\left( \pm 2{ }^{\circ} \mathrm{C}\right)$ and $16 \mathrm{~L}: 8 \mathrm{D}$ photoperiod. We then amplified the

452 fungal isolates through 2-3 rounds of inoculations to generate enough spores for the experiments

453 described below.

454

455 Common garden experiment. We performed the common garden experiment to test how

456 inoculating a single leaf of the rosette with a single parasite strain ("priming") affected

457 susceptibility of the plant to later-arriving parasite strains. This common garden experiment was

458 performed in a $30 \times 45 \mathrm{~m}$ fenced field at the University of Helsinki's Lammi Biological Station 
459 (Lammi, Finland). The experiment consisted of a total of 320 plants placed in groups of eight in 460 plastic trays $(0.5 \times 0.3 \mathrm{~m})$, with two plants from each of the four maternal plant lines in each of 46140 trays. Each tray was then assigned to one of four priming strains. Trays were equally spaced 462 apart in the field along a $5 \mathrm{~m}$ x $5 \mathrm{~m}$ grid. Each plant included two leaves from the same rosette 463 spiral enclosed in separate sleeves made from spore-proof polyester material (pollination bags 464 from PBS International) and secured at the leaf base, which were used for a separate study and 465 are not discussed further. One plant from each maternal line in the tray was assigned to the 466 "primed" treatment, and the other plants were assigned to the mock-inoculated "control" 467 treatment. On 26 July 2015, primed plants were enclosed in a plastic bag with a single leaf 468 emerging through a small hole in the bag. A fine paintbrush was used to inoculate that leaf with 469 one of the four priming strains, depending on the tray ID, so that each of the four priming strains 470 was represented 40 -times in the experiment ( 4 maternal lines per tray $\times 10$ trays per priming 471 strain). Twenty-four hours after inoculation, the leaf was covered with a spore-proof sleeve, the 472 plastic bag was wiped with ethanol and removed. Control plants underwent the same procedure, 473 but no powdery mildew was inoculated.

474 The plants in the common garden were then bulk-exposed to all four powdery mildew 475 strains, simultaneously, over the course of four days (30 July-2 August; days 4 to 8 post476 priming). This was done by rotating heavily infected source plants next to the trays, such that 477 each tray was exposed to each of the strains for 24 hours.

478 The plants in the common garden were screened for infection between 19-23 August. We 479 recorded the total number of uninfected and infected leaves for each plant. We collected several 480 infected leaves from each plant for genotyping. These infected leaves were stored in paper 481 envelopes at room temperature until DNA extraction (see Genetic analyses section below). 
484 priming influenced subsequent infection among sentinel plants placed into three field

485 populations during natural epidemics. We used the same set of four host plant maternal lines as

486 in the common garden experiment, and plants in the priming treatment were inoculated with a

487 parasite strain that had been present in the field population the previous year (Supplementary

488 Table 8). Priming of a single leaf was performed as in the common garden experiment. To

489 manipulate infection timing, plants were primed either 8 days (treatment P1) or 4 days (treatment

490 P2) prior to being placed into the three field populations on 4 August 2015. There were also C1

491 and C2 control plants set up at the same time (but mock inoculated). For each of the four host

492 plant maternal lines in each of the three field populations, we had 10 replicates of P1, 10

493 replicates of $\mathrm{P} 2,5$ replicates of $\mathrm{C} 1$, and 5 replicates of $\mathrm{C} 2$, for a total of 360 plants. Groups of

494 paired primed and control sentinel plants were placed on plastic trays throughout the field

495 populations. The trays were watered and moved to new locations every two days for 8 days, to

496 standardize exposure to powdery mildew spores. After the 8 days of exposure to natural

497 epidemics, the plants were covered with individual spore-proof pollination bags and transported

498 back to the Lammi Biological Station, where infections were allowed to continue developing for 499 another 10 days. Then we counted infected and uninfected leaves in each size class as described 500 for the common garden experiment. We also saved infected leaves in individual paper envelopes 501 to genotype and determine which strains infected them.

502 While the sentinels were in the field populations, we surveyed wild plants from each

503 population for infection to serve as a reference group for data visualization. We then tagged 504 infected wild plants located at least $1.5 \mathrm{~m}$ apart. Up to 44 infected plants per population were 
tagged. At the end of the epidemics, we collected samples of infected leaves from the tagged plants for genotyping and parasite strain identification. Infected leaves were stored in paper

507 envelopes at room temperature until prepared as samples. These samples were used as a

508 reference for data visualization, but were not included in any statistical model.

511 and consisted of fifteen host populations that had been infected for at least three consecutive

512 years ${ }^{97}$. Distances between pairs of populations ranged from $\sim 1 \mathrm{~km}$ to $\sim 40 \mathrm{~km}$. Each

513 population was surveyed biweekly for infection starting on 7 July, 2014, by visually scanning

514 plants for signs of the mildew. When host individuals became infected, the date of infection was

515 recorded and those hosts were physically tagged in order to be resurveyed as focal hosts. Up to

516 thirty focal hosts were tagged in each population. Focal plants were located at least $3 \mathrm{~m}$ from one

517 another and their locations were recorded by GPS. To minimize the impact of sampling on

518 pathogen community assembly, we sampled lesions from focal hosts and genotyped the

519 infections only after more than one leaf of a focal host was infected. We sampled lesions in such

520 a manner that spores also remained on the plants, thus the infection was not removed from the

521 epidemic. All hosts that survived were then resampled at the end of the season $(n=105$ hosts

522 across 13 populations). Sampling consisted of placing infected leaves in paper envelopes, which

523 were stored in a cool, dry place until the end of the field season, at which point the samples were

524 taken to Helsinki and stored at -20C. These two genotyping sessions were used to infer the 525 sequence of infection on host individuals, while the frequent surveys of whole host populations 526 were used to infer phenology of the parasite strains. 
Genetic analyses. We genotyped infections in all three studies to determine which

529 powdery mildew strains successfully established on the host plants. Each sample consisted of a

530 lesion from an infected leaf, which we placed into a $1.5 \mathrm{~mL}$ tube that was stored at $-20^{\circ} \mathrm{C}$ until

531 DNA was extracted using an E.Z.N.A. Plant Mini Kit (Omega Bio-Tek, Norcross, GA) at the

532 Institute of Biotechnology, University of Helsinki. The lesions consisted of both host tissue and

533 fungal material. Samples were genotyped at 19 single nucleotide polymorphism (SNP) loci with

534 the Sequenome iPlex platform at the Institute for Molecular Medicine Finland See 101,103 for details.

535 Because Po. plantaginis conidial spores are haploid, samples were classified as coinfected if two

536 different nucleotides were called at any locus ${ }^{101}$. The observed coinfections were resolved into

537 single infections with an algorithm that compared each coinfection profile to the genotypes of all

538 single infections in the experiment (i.e., to the four strains in the common garden experiment or

539 to all single infections from the same population in the natural epidemic experiment). When a

540 match was found, the genotype of the other coinfecting strain could be determined as having the

541 complementary alleles at the heterozygous loci. However, for samples with only a few

542 heterozygous loci and where multiple strains had the same nucleotides at those loci, we could

543 only unambiguously identify one of the two coinfecting strains. For samples from the common

544 garden experiment that failed to call all 19 SNPs, we were still able to identify the strain if the

545 nucleotides at the successfully called SNPs were unique to one of the four strains in that

546 experiment. However, samples from the natural epidemic experiment or wild host surveys that

547 were missing genotype data from any of the 19 SNPs were excluded from the analysis.

548 From sentinel plants in the common garden and natural epidemic experiments, we

549 randomly selected four infected leaves per plant for genotyping (if fewer than four leaves on the

550 plant were infected, then all infected leaves were sampled). In addition, we genotyped infections 
551 from a subset of the primed leaves to verify that plants were primed with the correct parasite

552 strains. A large number of samples failed to call several of the 19 SNP loci during our first round

553 of genotyping in spring 2016. To replace those samples for which genotyping failed, we

554 extracted DNA from remaining infected leaves from the same plants and genotyped those

555 replacement samples in spring 2017.

556

557 Analysis

$558 \quad$ All analyses were conducted in R version 3.5.2 ${ }^{104}$. We omitted plants from analyses that

559 were inoculated, but never became infected or that were mock-inoculated but became infected

560 (85 in the common garden experiment; 165 in the natural epidemic experiment), as well as plants

561 that died prior to data collection (4 in the common garden experiment; 2 in the natural epidemic

562 experiment) resulting in a total sample size of $n=231$ in the common garden experiment and $n=$

563193 in the natural epidemic experiment.

$564 \quad$ Common garden experiment. We first tested whether the priming treatment altered

565 infection during the common garden by constructing three models using the R package lme4 ${ }^{105}$ :

566 (1) the probability of a plant becoming infected, using a logistic mixed model, (2) the logit-

567 transformed proportion of leaves infected as a response measure representing infection severity,

568 using a linear mixed model, and (3) the logit-transformed proportion of leaves infected as a

569 response measure representing infection severity, limited to infected hosts only, using a linear

570 mixed model. All three models included the experimental treatment (inoculated vs. mock

571 inoculated) as a fixed effect. We included the log-transformed number of leaves as a fixed

572 covariate in the model, because plants with more leaves have a higher probability of intercepting

573 infectious spores. Inoculation tray was included as a random effect in the model. To test whether 
574 treatment effects differed among host genotypes and priming strains, we fit three models with the

575 same three response variables, this time including the full priming treatment (five levels: mock-

576 inoculated, and four priming strains), plant genotype, and their interactions as fixed effects, the

577 log-transformed total number of leaves as a fixed covariate, and priming tray as a random effect.

578 We evaluated differences among various model coefficients using the emmeans package in $\mathrm{R}^{106}$.

579 To test whether the priming strain affected other later arriving strains at least as strongly

580 as it affected itself, we fit a model with a binomial response representing whether or not the host

581 became infected, and including the priming treatment as a binary factor (control vs primed) and

582 its interaction with whether or not the infecting strain was different from the priming strain. The

583 model also included log-transformed total number of leaves as a fixed covariate and the tray and

584 plant id as nested random intercepts. Finally, we tested whether within-host priority effects

585 altered the structure of parasite assemblages, using a multivariate generalized linear model using

586 the MVabun package in $\mathrm{R}^{62}$. To measure the effect of prior infection on the structure parasite

587 assemblages, we constructed a model that tests whether the distribution of infection by each

588 strain was affected by the priming treatment (five levels: control, and four priming genotypes),

589 plant genotype, and their interactions, including the log-transformed total number of leaves as a

590 covariate. These models avoid some of the problems associated with distance-based models such

591 as permANOVA. However, because these models cannot handle unbalanced grouping variables,

592 experimental tray was included as a covariate rather than a random effect in the model.

$593 \quad$ Natural epidemic experiment. We first tested whether the priming treatment altered

594 infection during the natural epidemic by constructing three models using the lme4 package in R:

595 (1) the probability of a plant becoming infected, using a logistic mixed model, (2) the logit-

596 transformed proportion of leaves infected as a response measure representing infection severity, 
597 using a linear mixed model, and (3) the logit-transformed proportion of leaves infected as a 598 response measure representing infection severity, limited to infected hosts only, using a linear 599 mixed model. Each model included host population, plant genotype, and the experimental 600 treatment $(\mathrm{C} 1$ : control for the first priming treatment, $\mathrm{C} 2$ : control for the second priming 601 treatment, P1: first priming treatment at eight days prior to placement in the field, and P2: second 602 priming treatment at four days prior to placement in the field) as interactive fixed effects and the 603 log-transformed total number of leaves as a fixed covariate. Experimental tray was included as a 604 random intercept in the model, but this random effect did not explain any of the variance in some 605 of the models, leading to a computational singularity. Despite the computational singularity, we 606 opted to keep experimental tray in all models to account for non-independence among samples in 607 each patch. In the logistic regression model, there were no significant interactions $(\mathrm{p}=1)$, and 608 owing to the binary response variable and high number of predictors, the model suffered from 609 complete separation. We therefore iteratively removed all non-significant interactions, yielding a 610 reduced model. We evaluated differences among various model coefficients in the reduced 611 models using the emmeans package.

612 We tested whether priority effects among parasite strains could lead to variation in the 613 structure of parasite assemblages among hosts during a natural epidemic with a multivariate 614 generalised linear model using the MVabun package in $\mathrm{R}$, to test whether the distribution of 615 infection by each strain was affected by the priming treatment (four levels: C1, C2, P1, and P2), 616 patch, plant genotype, and their interactions, including the total number of leaves as a covariate. 617 To fit this model, we removed from the dataset any plant that had an infection that could not be 618 genotyped, resulting in a total of 181 plants for this analysis. Similar to previous analyses, we 619 reduced the model by removing non-significant interactions. To visualize these results, we 
620 performed an unconstrained ordination using the boral package in $\mathrm{R}^{107}$, and then plotted the 621 results using the ggboral package in $\mathrm{R}^{108}$.

622 Wild host survey. To test whether parasite strains exhibit priority effects within hosts, we

623 first fit a series of cox proportional hazards models following Halliday et al ${ }^{18,36}$ using the coxphf

624 package in $\mathrm{R}^{109}$. To fit these models, we made one critical assumption about the data: we

625 assumed that whichever strain was first observed in genotyping was the "early arriving strain".

626 This assumption allows us to use temporal data from the first observations of a host, regardless

627 of the time between that survey and the genotyping date. However, this assumption ignores the 628 possibility of rare parasite strains being locally cleared early during the epidemic. Furthermore, 629 because we could not resolve the sequence of infection on host individuals that are coinfected 630 during the initial genotyping survey (occurring in 22/105 host individuals), both coinfecting 631 parasites were assumed to have been present at the initial infection of the host. We then fit a 632 series of cox proportional hazards models of infection by each parasite as a focal (i.e., late633 arriving) parasite with prior infection by each other (i.e., early-arriving) parasite as the only

634 predictor in each model. These models tested whether the time until infection by each strain was 635 influenced by infection sequence on a host individual. Across the Åland archipelago, distinct 636 host populations often harbor distinct parasite assemblages. Parasite strains were therefore only 637 modeled among hosts that occurred in populations where those Parasites had been observed. To 638 explore whether the magnitude of priority effects among parasite strains tended to be facilitative 639 or antagonistic, we next fit an intercept only linear mixed model with the coefficient from the 640 cox proportional hazards models (i.e., the interaction coefficient) as the response variable and the 641 identity of the focal (i.e., late arriving) parasite in the cox proportional hazards models as a 642 random intercept, weighting the regression by the number of surveys per host individual to give 
643 more explanatory power to host individuals that were surveyed more times over the growing

644 season. Finally, we tested whether parasite phenology among strains altered the structure of

645 parasite assemblages within hosts using a multivariate generalised linear model on the

646 distribution of infections by each strain at the end of the epidemic, in the R package MVabund.

647 To measure parasite phenology, we recorded the earliest date that each strain was observed in the

648 field during the 2014 epidemic. We then modeled the presence or absence of each strain at the

649 end of the season as a function of the phenology of the early-infecting strains, with the sampling

650 date of the final survey and host population as covariates in the model.

651

652 Data availability statement: The data supporting the results are archived on Figshare (DOI:

$653 \quad 10.6084 / \mathrm{m} 9$. figshare.12627806)

654

655 Code availability statement: The code supporting the results is archived on Figshare (DOI:

$656 \quad 10.6084 / \mathrm{m} 9$. figshare.12627806) 


\section{References}

658 1. Dobson, A., Lafferty, K. D., Kuris, A. M., Hechinger, R. F. \& Jetz, W. Homage to 659 Linnaeus: How many parasites? How many hosts? Proc. Natl. Acad. Sci. 105, 11482$66011489(2008)$.

661 2. Mideo, N. Parasite adaptations to within-host competition. Trends Parasitol. 25, 261-8 662 (2009).

663 3. Greischar, M. A. et al. Evolutionary consequences of feedbacks between within-host 664 competition and disease control. Evol. Med. Public Heal. 2020, 30-34 (2020).

665 4. Wale, N. et al. Resource limitation prevents the emergence of drug resistance by 666 intensifying within-host competition. Proc. Natl. Acad. Sci. U. S. A. 114, 13774-13779 667 (2017).

668 5. Bhattacharya, A., Toro Díaz, V. C., Morran, L. T. \& Bashey, F. Evolution of increased 669 virulence is associated with decreased spite in the insect-pathogenic bacterium 670 Xenorhabdus nematophila. Biol. Lett. 15, 20190432 (2019).

671 6. Susi, H., Barrès, B., Vale, P. F. \& Laine, A.-L. Co-infection alters population dynamics of 672 infectious disease. Nat. Commun. 6, 5975 (2015).

673 7. Read, A. F. \& Taylor, L. H. The ecology of genetically diverse infections. Science 292, 674 1099-102(2001).

675 8. Hawley, D. M. \& Altizer, S. M. Disease ecology meets ecological immunology:

676 understanding the links between organismal immunity and infection dynamics in natural 677 populations. Funct. Ecol. 25, 48-60 (2011).

678 9. Hoverman, J. T., Hoye, B. J. \& Johnson, P. T. J. Does timing matter? How priority effects 679 influence the outcome of parasite interactions within hosts. Oecologia 173, 1471-1480 
(2013).

681 10. Zhan, J. \& McDonald, B. a. Experimental measures of pathogen competition and relative 682 fitness. Annu. Rev. Phytopathol. 51, 131-53 (2013).

683 11. Hellard, E., Fouchet, D., Vavre, F. \& Pontier, D. Parasite-Parasite Interactions in the 684 Wild: How To Detect Them? Trends in Parasitology vol. 31 640-652 (2015).

685 12. Tollenaere, C., Susi, H. \& Laine, A. L. Evolutionary and Epidemiological Implications of 686 Multiple Infection in Plants. Trends Plant Sci. 21, 80-90 (2015).

687 13. Budischak, S. A. et al. Competing for blood: the ecology of parasite resource competition 688 in human malaria-helminth co-infections. Ecol. Lett. 21, 536-545 (2018).

689 14. Griffiths, E. C., Pedersen, A. B., Fenton, A. \& Petchey, O. L. Analysis of a summary 690 network of co-infection in humans reveals that parasites interact most via shared resources. Proc. R. Soc. B Biol. Sci. 281, 20132286-20132286 (2014).

692 15. Ezenwa, V. O. Helminth-microparasite co-infection in wildlife: lessons from ruminants, 693 rodents and rabbits. Parasite Immunol. 38, 527-534 (2016).

694 16. Lello, J., Boag, B., Fenton, A., Stevenson, I. R. \& Hudson, P. J. Competition and 695 mutualism among the gut helminths of a mammalian host. Nature 428, 840-844 (2004).

696 17. Chung, E., Petit, E., Antonovics, J., Pedersen, A. B. \& Hood, M. E. Variation in resistance 697

698 to multiple pathogen species: anther smuts of Silene uniflora. Ecol. Evol. 2, 2304-14 (2012).

699 18. Halliday, F. W., Umbanhowar, J. \& Mitchell, C. E. A host immune hormone modifies 700 parasite species interactions and epidemics: insights from a field manipulation. Proc. $R$.

701 Soc. B Biol. Sci. 285, 20182075 (2018).

702 19. Eswarappa, S. M., Estrela, S. \& Brown, S. P. Within-host dynamics of multi-species 
infections: Facilitation, competition and virulence. PLoS ONE vol. 7 e38730 (2012).

704 20. Zélé, F., Magalhães, S., Kéfi, S. \& Duncan, A. B. Ecology and evolution of facilitation 705 among symbionts. Nat. Commun. 9, 4869 (2018).

706 21. Jenner, E. An Inquiry into the Causes and Effects of the Variolae Vaccinae, a Disease 707 Discovered in Some of the Western Countries of England, Particularly Gloucestershire, 708 and Known by the Name of "The Cow Pox" (1798). Reprinted by Milan: R Lier \& Co vol. 70984 (Milan, R. Lier \& Co., 1923).

710 22. Fulton, R. W. Practices and Precautions in the Use of Cross Protection for Plant Virus 711 Disease Control. Annu. Rev. Phytopathol. 24, 67-81 (1986).

712 23. Van Loon, L. C. Induced resistance in plants and the role of pathogenesis-related proteins. 713 Eur. J. Plant Pathol. 103, 753-765 (1997).

714 24. Conrath, U. et al. Priming: Getting Ready for Battle. Mol. Plant-Microbe Interact. 19, $715 \quad 1062-1071(2006)$.

716 25. Pieterse, C. M. J. et al. Induced Systemic Resistance by Beneficial Microbes. Annu. Rev. $717 \quad$ Phytopathol. 52, 347-375 (2014).

718 26. Spoel, S. H., Johnson, J. S. \& Dong, X. Regulation of tradeoffs between plant defenses 719 against pathogens with different lifestyles. Proc. Natl. Acad. Sci. U. S. A. 104, 18842-7 $720 \quad$ (2007).

721 27. Kliebenstein, D. J. \& Rowe, H. C. Ecological costs of biotrophic versus necrotrophic 722 pathogen resistance, the hypersensitive response and signal transduction. Plant Sci. 174, $723 \quad 551-556(2008)$.

724 28. Koornneef, A. et al. Kinetics of Salicylate-Mediated Suppression of Jasmonate Signaling 725 Reveal a Role for Redox Modulation. Plant Physiol. 147, 1358-1368 (2008). 
726

727

728

729

730

731

732

733

734

735

736

737

738

739

740

741

742

743

744

745

746

747

748

29. Glazebrook, J. Contrasting Mechanisms of Defense Against Biotrophic and Necrotrophic Pathogens. Annu. Rev. Phytopathol. 43, 205-227 (2005).

30. Ezenwa, V. O., Etienne, R. S., Luikart, G., Beja-Pereira, A. \& Jolles, A. E. Hidden consequences of living in a wormy world: nematode-induced immune suppression facilitates tuberculosis invasion in African buffalo. Am. Nat. 176, 613-24 (2010).

31. Clay, P. A., Cortez, M. H., Duffy, M. A. \& Rudolf, V. H. W. Priority effects within coinfected hosts can drive unexpected population-scale patterns of parasite prevalence. Oikos 128, 571-583 (2019).

32. Clay, P. A., Duffy, M. A. \& Rudolf, V. H. W. Within-host priority effects and epidemic timing determine outbreak severity in co-infected populations. Proc. R. Soc. B Biol. Sci. 287, 20200046 (2020).

33. Clark, P., Ward, W., Lang, S., Saghbini, A. \& Kristan, D. Order of Inoculation during Heligmosomoides bakeri and Hymenolepis microstoma Coinfection Alters Parasite Life History and Host Responses. Pathogens 2, 130-152 (2013).

34. Fukami, T. Historical contingency in community assembly : integrating niches, species pools, and priority effects. Annu. Rev. Ecol. Evol. Syst. 46, 1-23 (2015).

35. Vannette, R. L. \& Fukami, T. Historical contingency in species interactions: Towards niche-based predictions. Ecol. Lett. 17, 115-124 (2014).

36. Halliday, F. W., Umbanhowar, J. \& Mitchell, C. E. Interactions among symbionts operate across scales to influence parasite epidemics. Ecol. Lett. 20, 1285-1294 (2017).

37. Johnson, P. T. J., de Roode, J. C. \& Fenton, A. Why infectious disease research needs community ecology. Science (80-. ). 349, 1259504 (2015).

38. Karvonen, A., Jokela, J. \& Laine, A.-L. Importance of Sequence and Timing in Parasite 
Coinfections. Trends Parasitol. 35, 109-118 (2019).

750 39. Mordecai, E. A., Gross, K. \& Mitchell, C. E. Within-Host Niche Differences and Fitness Trade-offs Promote Coexistence of Plant Viruses. Am. Nat. 187, E13-E26 (2016).

752 40. Kuris, A. M., Blaustein, A. R. \& Alio, J. J. Hosts as Islands. Am. Nat. 116, 570-586

$753 \quad(1980)$

754 41. Rynkiewicz, E. C., Pedersen, A. B. \& Fenton, A. An ecosystem approach to understanding 755 and managing within-host parasite community dynamics. Trends Parasitol. 31, 212-221

756 (2015).

757 42. Sousa, W. P. Interspecific Interactions among Larval Trematode Parasites of Freshwater and Marine Snails Interspecific Interactions Among Larval Trematode Parasites of Freshwater and Marine Snails. Am. Zool. 32, 583-592 (1992).

760

43. Graham, A. L. Ecological rules governing helminth microparasite coinfection. Proc. Natl. Acad. Sci. 105, 566-570 (2008).

762 44. Seabloom, E. W. et al. The community ecology of pathogens: Coinfection, coexistence and community composition. Ecol. Lett. 18, 401-415 (2015).

764 45. Cobey, S. \& Lipsitch, M. Pathogen diversity and hidden regimes of apparent competition.

765 Am. Nat. 181, 12-24 (2013).

766 46. Greischar, M. A. \& Koskella, B. A synthesis of experimental work on parasite local 767 adaptation. Ecol. Lett. 10, 418-434 (2007).

768 47. Hoeksema, J. D. \& Forde, S. E. A meta-analysis of factors affecting local adaptation 769 between interacting species. Am. Nat. 171, 275-290 (2008).

770 48. Burdon, J. J. \& Laine, A.-L. Evolutionary dynamics of plant pathogen interactions. $771 \quad$ (Cambridge University Press, 2019). 
772 49. Lambrechts, L., Fellous, S. \& Koella, J. C. Coevolutionary interactions between host and 773 parasite genotypes. Trends Parasitol. 22, 12-16 (2006).

774 50. Ferro, K. et al. Experimental evolution of immunological specificity. Proc. Natl. Acad. 775 Sci. U. S. A. 116, 20598-20604 (2019).

776 51. Westman, S. M., Kloth, K. J., Hanson, J., Ohlsson, A. B. \& Albrectsen, B. R. Defence 777 priming in Arabidopsis - a Meta-Analysis. Sci. Rep. 9, 13309 (2019).

778 52. Pedersen, A. B. \& Fenton, A. Emphasizing the ecology in parasite community ecology. $779 \quad$ Trends Ecol. \&amp; Evol. 22, 133-139 (2007).

780 53. Pedersen, A. B. \& Fenton, A. The role of antiparasite treatment experiments in assessing 781 782 the impact of parasites on wildlife. Trends Parasitol. 31, 200-11 (2015).

54. Laine, A. L. Context-dependent effects of induced resistance under co-infection in a plantpathogen interaction. Evol. Appl. 4, 696-707 (2011).

$78455 . \quad$ Conrath, U., Beckers, G. J. M., Langenbach, C. J. G. \& Jaskiewicz, M. R. Priming for 785 Enhanced Defense. Annu. Rev. Phytopathol. 53, 97-119 (2015).

786 56. Douma, J. C., Vermeulen, P. J., Poelman, E. H., Dicke, M. \& Anten, N. P. R. When does 787 it pay off to prime for defense? A modeling analysis. New Phytol. 216, 782-797 (2017).

788 57. Mauch-Mani, B., Baccelli, I., Luna, E. \& Flors, V. Defense Priming: An Adaptive Part of 789 Induced Resistance. Annu. Rev. Plant Biol. 68, 485-512 (2017).

790 58. Budischak, S. A. et al. Resource limitation alters the consequences of co-infection for 791 both hosts and parasites. Int. J. Parasitol. 45, 455-463 (2015).

792 59. Borer, E. T., Laine, A.-L. \& Seabloom, E. W. A multiscale approach to plant disease 793 using the metacommunity concept. Annu. Rev. Phytopathol. 54, 397-418 (2016).

794 60. Bushnell, W. R. The role of powdery mildew research in understanding host-parasite 
interactions. in The powdery mildews: a comprehensive treatise (eds. Belanger, R. R., Bushnell, W. R., Dik, A. J. \& Carver, T. L. W.) 1-12 (APS Press, 2002).

61. Warton, D. I., Wright, S. T. \& Wang, Y. Distance-based multivariate analyses confound location and dispersion effects. Methods Ecol. Evol. 3, 89-101 (2012).

62. Wang, Y., Naumann, U., Wright, S. T. \& Warton, D. I. Mvabund- an R package for model-based analysis of multivariate abundance data. Methods Ecol. Evol. 3, 471-474

801 (2012).

802

803

804

63. Benesh, D. P. \& Kalbe, M. Experimental parasite community ecology: intraspecific variation in a large tapeworm affects community assembly. J. Anim. Ecol. 85, 1004-1013 (2016).

805

64. Mucha, J. et al. Effect of Simulated Climate Warming on the Ectomycorrhizal Fungal Community of Boreal and Temperate Host Species Growing Near Their Shared Ecotonal Range Limits. Microb. Ecol. 75, 348-363 (2018).

808

65. Chang, A. L., Brown, C. W., Crooks, J. A. \& Ruiz, G. M. Dry and wet periods drive rapid

811 66. David, A. S., Seabloom, E. W. \& May, G. Disentangling environmental and host sources of fungal endophyte communities in an experimental beachgrass study. Mol. Ecol. 26,

814 67. Penczykowski, R. M., Parratt, S. R., Barrès, B., Sallinen, S. K. \& Laine, A. L. 815 Manipulating host resistance structure reveals impact of pathogen dispersal and

817 68. Pieterse, C. M. J., Van der Does, D., Zamioudis, C., Leon-Reyes, A. \& Van Wees, S. C. 
M. Hormonal modulation of plant immunity. Annu. Rev. Cell Dev. Biol. 28, 489-521

819 (2012).

820

69. Susi, H. \& Laine, A.-L. The effectiveness and costs of pathogen resistance strategies in a 821 perennial plant. J. Ecol. 103, 303-315 (2015).

822 70. Höckerstedt, L. Evolutionary and ecological dimensions of disease resistance. Thesis at: 823 https://helda.helsinki.fi/handle/10138/314983 (2020).

824 71. Macke, E. et al. Diet and Genotype of an Aquatic Invertebrate Affect the Composition of 825 Free-Living Microbial Communities. Front. Microbiol. 11, 380 (2020).

826 72. Wagner, M. R. et al. Host genotype and age shape the leaf and root microbiomes of a wild perennial plant. Nat. Commun. 7, 12151 (2016).

828 73. Koch, H. \& Schmid-Hempel, P. Socially transmitted gut microbiota protect bumble bees 829 against an intestinal parasite. Proc. Natl. Acad. Sci. 108, 19288-19292 (2011).

830 74. Biere, A. \& Goverse, A. Plant-Mediated Systemic Interactions Between Pathogens, 831 Parasitic Nematodes, and Herbivores Above-and Belowground. Annu. Rev. Phytopathol 54, 499-527 (2016).

833 75. Little, T. J., Watt, K. \& Ebert, D. Parasite-Host Specificity: Experimental Studies on the Basis of Parasite Adaptation. Evolution (N. Y). 60, 31 (2006).

835 76. Seybold, H. et al. A fungal pathogen induces systemic susceptibility and systemic shifts in 836 wheat metabolome and microbiome composition. Nat. Commun. 11, 1910 (2020).

837 77. Cui, J. et al. Pseudomonas syringae manipulates systemic plant defenses against 838 pathogens and herbivores. Proc. Natl. Acad. Sci. U. S. A. 102, 1791-1796 (2005).

839 78. Mideo, N., Alizon, S. \& Day, T. Linking within- and between-host dynamics in the 840 evolutionary epidemiology of infectious diseases. Trends Ecol. \&amp; Evol. 23, 511-517 
(2008).

842 79. Pedersen, A. B. \& Greives, T. J. The interaction of parasites and resources cause crashes in a wild mouse population. J. Anim. Ecol. 77, 370-7 (2008).

844 80. Laine, A.-L., Barrès, B., Numminen, E. \& Siren, J. P. Variable opportunities for 845 846 outcrossing result in hotspots of novel genetic variation in a pathogen metapopulation.

847

81. Vaumourin, E. \& Laine, A.-L. Role of Temperature and Coinfection in Mediating Pathogen Life-History Traits. Front. Plant Sci. 9, 1670 (2018).

82. Numminen, E., Vaumourin, E., Parratt, S. R., Poulin, L. \& Laine, A.-L. Variation and correlations between sexual, asexual and natural enemy resistance life-history traits in a natural plant pathogen population. BMC Evol. Biol. 19, 142 (2019).

852 83. Tack, A. J. M., Thrall, P. H., Barrett, L. G., Burdon, J. J. \& Laine, A. -L. Variation in 853 infectivity and aggressiveness in space and time in wild host-pathogen systems: causes and consequences. J. Evol. Biol. 25, 1918-1936 (2012).

84. Penczykowski, R. M., Laine, A. L. \& Koskella, B. Understanding the ecology and 856 evolution of host-parasite interactions across scales. Evol. Appl. 9, 37-52 (2016).

857 85. Rynkiewicz, E. C., Fenton, A. \& Pedersen, A. B. Linking community assembly and 858 structure across scales in a wild mouse parasite community. Ecol. Evol. 9, 13752-13763 (2019).

860 86. Bolnick, D. I. et al. Why intraspecific trait variation matters in community ecology. 861 Trends Ecol. Evol. 26, 183-92 (2011).

862 87. Siefert, A. Incorporating intraspecific variation in tests of trait-based community 863 assembly. Oecologia 170, 767-775 (2012). 
864 88. Laughlin, D. C. et al. A predictive model of community assembly that incorporates intraspecific trait variation. Ecol. Lett. 15, 1291-9 (2012).

89. Shaw, D. J. \& Dobson, A. P. Patterns of macroparasite abundance and aggregation in wildlife populations: a quantitative review. Parasitology 111, S111-S133 (1995).

868

90. Lloyd-Smith, J. O., Schreiber, S. J., Kopp, P. E. \& Getz, W. M. Superspreading and the effect of individual variation on disease emergence. Nature 438, 355-359 (2005).

91. Sagar, G. R. \& Harper, J. L. Plantago major L., P. media L. and P. lanceolata. J. Ecol. 52, 871 189-221 (1964).

872 92. Ross, M. D. Inheritance of self-incompatibility in Plantago lanceolata. Heredity (Edinb).

873 30, 169-176 (1973).

874 93. Ojanen, S. P., Nieminen, M., Meyke, E., Pöyry, J. \& Hanski, I. Long-term metapopulation 875 study of the Glanville fritillary butterfly (Melitaea cinxia): Survey methods, data management, and long-term population trends. Ecol. Evol. 3, 3713-3737 (2013).

877 94. Tollenaere, C. \& Laine, A. L. Investigating the production of sexual resting structures in a 878 plant pathogen reveals unexpected self-fertility and genotype-by-environment effects. $J$. 879 Evol. Biol. 26, 1716-1726 (2013).

880 95. Tack, A. \& Laine, A. Ecological and evolutionary implications of spatial heterogeneity 881 during the off-season for a wild plant pathogen. New Phytol. 65, 297-308 (2014).

882 96. Laine, A. L. \& Hanski, I. Large-scale spatial dynamics of a specialist plant pathogen in a 883 fragmented landscape. J. Ecol. 94, 217-226 (2006).

884 97. Jousimo, J. et al. Ecological and evolutionary effects of fragmentation on infectious 885 disease dynamics. Science (80-. ). 344, 1289-1293 (2014).

886 98. Laine, A. L. Resistance variation within and among host populations in a plant-pathogen 
metapopulation: Implications for regional pathogen dynamics. J. Ecol. 92, 990-1000 (2004).

99. Penczykowski, R. M., Walker, E., Soubeyrand, S. \& Laine, A.-L. Linking winter conditions to regional disease dynamics in a wild plant-pathogen metapopulation. New

891 Phytol. 205, 1142-1152 (2015).

892

100. Laine, A. L. Pathogen fitness components and genotypes differ in their sensitivity to nutrient and temperature variation in a wild plant-pathogen association. J. Evol. Biol. 20, $2371-2378$ (2007).

895

101. Tollenaere, C. et al. SNP Design from 454 Sequencing of Podosphaera plantaginis Transcriptome Reveals a Genetically Diverse Pathogen Metapopulation with High Levels of Mixed-Genotype Infection. PLoS One 7, e52492 (2012).

898

102. Nicot, P. C., Bardin, M. \& Dik, A. J. Basic methods for epidemiological studes of 899 powdery mildews: culture and preservation of isolates, production and delivery of 900 inoculum, and disease assessment. in The powdery mildews: a comprehensive treatise (eds. Belanger, R. R., Bushnell, W. R., Dik, A. J. \& Carver, T. L. W.) 83-99 (APS Press, 2002).

103. Parratt, S. R., Barrès, B., Penczykowski, R. M. \& Laine, A.-L. Local adaptation at higher trophic levels: contrasting hyperparasite-pathogen infection dynamics in the field and laboratory. Mol. Ecol. 26, 1964-1979 (2017).

906 104. R Core Team. R: a language and environment for statistical computing | GBIF.ORG. 907 (2015) doi:10.1007/978-3-540-74686-7.

908 909

using lme4. J. Stat. Softw. 67, 1-48 (2014). 
910

911

912

913

914

915

916

917

918

919

920

921

922

923

924 at the University of Helsinki are acknowledged for carrying out the DNA extractions and for

925 genotyping samples, respectively. This work was supported by the University of Zürich and by

926 grants from the Academy of Finland (296686) to A-LL and the European Research Council

927 (Consolidator Grant RESISTANCE 724508) to A-LL.

929 Author contributions: RMP and A-LL designed and implemented the experiments. BB

930 conducted the wild host survey. JLE compiled the wild host survey data. EN and RMP resolved

931 the parasite multi-locus genotypes. FWH analyzed the data and wrote the first draft. All authors

932 contributed substantially to revising the manuscript. 
934 Competing interests: We have no competing interests

936 Figure Legends:

937 Figure 1. Illustration of key differences and similarities among the three field studies presented

938 in this manuscript. Pot color represents different priming treatments $($ black $=$ control; blue $=$ 939 primed). Star color represents different parasite genotypes (i.e., strains). A) To test whether host

940 (i.e., plant) responses to prior infection can drive parasite assembly via priority effects, focal 941 potted hosts (black) were either inoculated or mock-inoculated with one of four priming strains.

942 Then hosts were exposed to all four priming strains by placing heavily infected potted hosts

943 (grey) adjacent to the focal hosts in a common garden, outdoors. This experiment included four

944 different host genotypes not depicted in this figure. B) To test whether host-mediated priority

945 effects among parasites can influence parasite assembly during a natural epidemic, focal potted 946 hosts (black) were either inoculated or mock inoculated with a priming strain associated with a 947 given host population and then embedded in a wild host population associated with that strain 948 (grey) during a natural epidemic. This experiment included four different host genotypes, two 949 infection timing treatments, and three host populations that are not depicted in this figure. C) To 950 test whether a signal of host-mediated priority effects among parasites could be detected in 951 natural host and parasite populations, infection on focal wild hosts (black) occurring in wild host 952 populations (grey) were repeatedly surveyed over time. This survey included 13 host populations 953 that are not depicted in this figure.

955 Figure 2. Results from the common garden experiment. The effect of the priming treatment on 
956 a) whether or not a host became infected with any strain; b) whether or not a host became

957 infected with the priming strain assigned to the experimental block (control hosts were mock-

958 inoculated with the priming strain associated with that block), or any non-priming strain; c)

959 whether or not a host became infected with any strain as a function of the priming strain, shown

960 as a log-response ratio; d) the percent of hosts infected by each strain during the common garden

961 experiment. In panels a-c, filled points are model-estimated means, error bars are model-

962 estimated $95 \%$ confidence intervals, and open points show the raw data. In panel d, points show

963 the percent of hosts infected and the error bars show one standard error around those points. In

964 total there were 132 control hosts and 99 primed hosts, of which 28 were primed with Strain

965 G46, 20 were primed with Strain O10, 28 were primed with Strain O15, and 23 were primed

966 with Strain O49. These results highlight the differential effect of each strain on the priming

967 response of the host as well as the differential sensitivity of each strain to that priming effect.

968 Only strain O15 was a uniformly poor primer of subsequent infection. All other parasite strains

969 significantly facilitated subsequent infection by at least one other strain. Together, these results

970 suggest that by differentially determining the host response to infection and experiencing

971 differential sensitivity to that response, prior infection can strongly alter assembly of these

972 parasite strains.

973

974 Figure 3. Results from the natural epidemic experiment. The effect of the sequence and timing

975 of infection on a) whether or not a host became infected with any strain, shown as a log-response

976 ratio between each control and each priming treatment. (e.g., $\mathrm{C} 1$ vs $\mathrm{P} 1$ and $\mathrm{C} 2$ vs $\mathrm{P} 2$ ); and b) the

977 number of hosts infected by each strain grouped by whether a host was experimental $(\mathrm{C} 1, \mathrm{C} 2$,

978 P2, P2) or wild (shown in grey). Plants were primed either 8 days (treatment P1) or 4 days 
979 (treatment P2) prior to being placed into the field. There were also C1 and C2 control plants set

980 up at the same time (but mock inoculated). Points are model-estimated means, and error bars are

981 model-estimated 95\% confidence intervals. Colors represent different strains, with pink and

982 orange corresponding to the two priming strains used in this experiment (O49 and Priming294,

983 respectively). These results show that, consistent with the common garden experiment,

984 experimental priming increased the probability of a host becoming infected. However, that effect

985 did not depend on infection timing (P1 vs P2). As a consequence of this effect, experimentally

986 primed hosts had more complex parasite communities that were more similar to wild hosts than

987 mock-inoculated control hosts.

989 Figure 4. Results from the wild host survey. a) The effect of infection sequence on the risk of a 990 host becoming infected with each parasite strain. The x-axis shows the focal (i.e., late arriving)

991 parasite strains from field-collected samples, arranged by their phenology. The grey, blue, and 992 red points are coefficient estimates from cox proportional hazards models measuring the pairwise 993 interaction between each focal strain and each co-occurring other (i.e., early arriving) strain; 994 point size represents the average number of surveys per host individual; blue and red indicate 995 significant facilitation and antagonism $(\mathrm{p}<0.05)$, respectively, and grey indicates insufficient 996 evidence for significant priority effects $(p>0.05)$. The colored points with error bars show the 997 mean and one standard error across all coefficients for each focal parasite strain. The black point 998 is the model estimated mean across all parasite strains, and the black error bar (which is small 999 and largely obscured by the black circle) is the $95 \%$ confidence interval from an intercept-only 1000 model, indicating that on average, interactions tended to be positive. b) The number of hosts 1001 infected by each strain at the end of the growing season. The x-axis shows hosts that became 
1002 infected ordered according to time when the first strain to infect those hosts (i.e., the early

1003 arriving strain) was first detected in the survey. The y-axis shows the abundance of all strains

1004 that accumulated on these hosts. Panels represent different host populations. Colors are arranged

1005 in order of parasite phenology from dark to light. Together, these results show that, across all

1006 parasite strains, interactions were most commonly facilitative (Panel a), the strength of

1007 facilitation increased slightly with phenology of the late-arriving strain (Panel a), and hosts that

1008 were first infected by strains with early phenology were ultimately infected with a more diverse

1009 assemblage of parasite strains by the end of the growing season (Panel b).

1010 
A) Common garden experiment:

Can the host response to prior infection drive parasite assembly via priority effects?

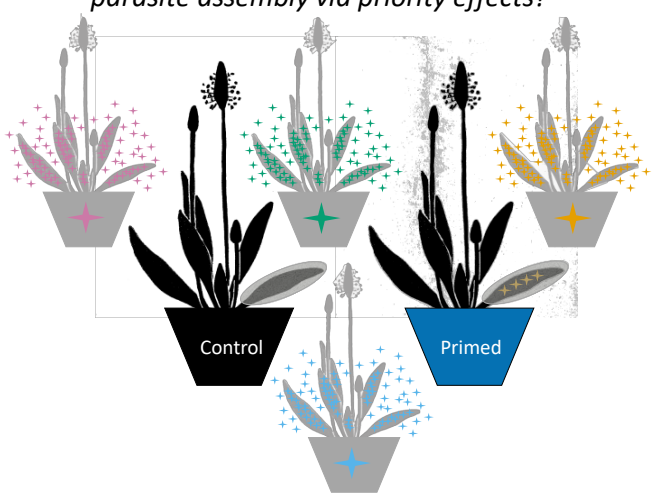

B) Natural epidemic experiment:

Can host-mediated priority effects influence parasite assembly during a natural epidemic?

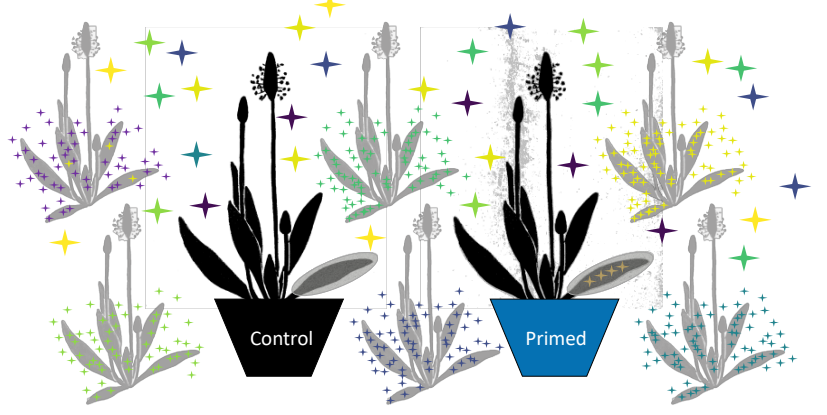

C) Wild host survey:

Can host-mediated facilitative priority effects be detected in natural populations?

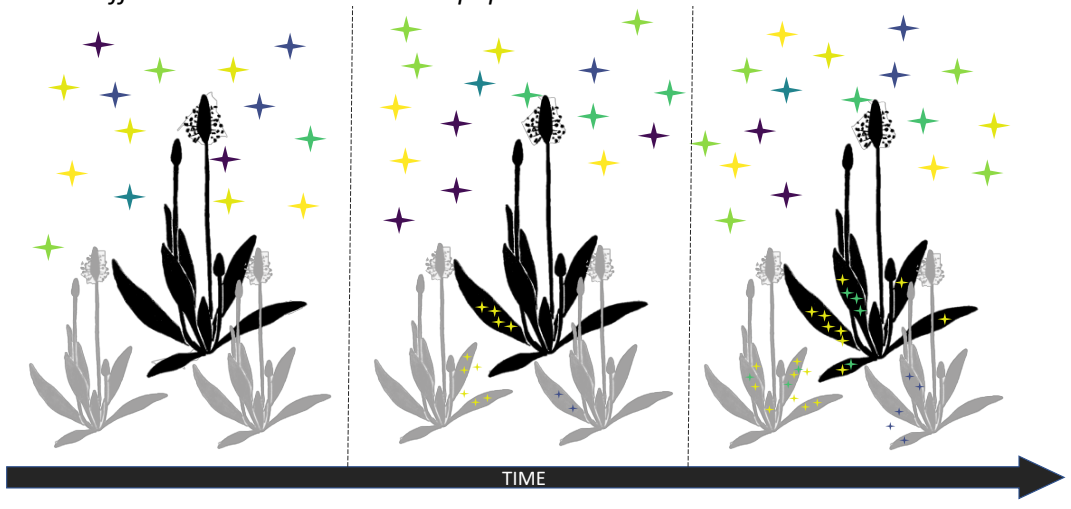



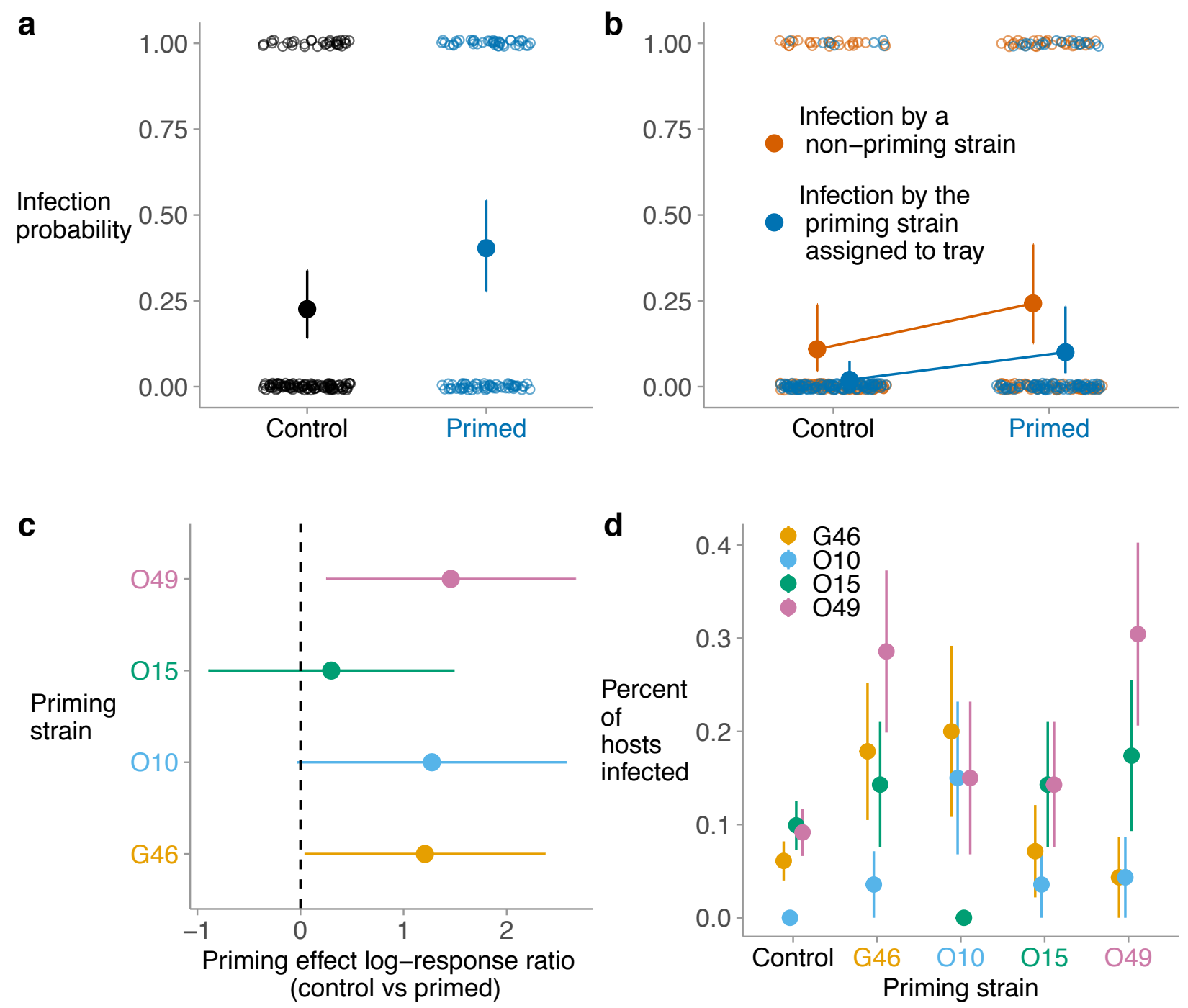

\section{$1014 \quad$ Figure 2}




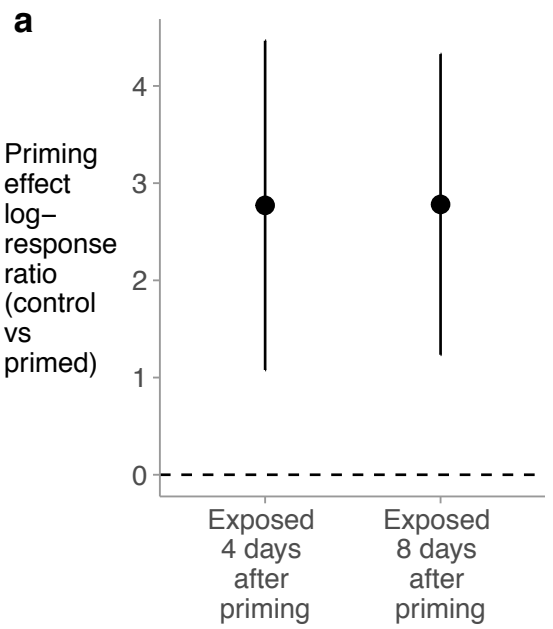

2015_MLG_100 2015_MLG_101

2015 MLG 104

2015 MLG $^{-109}$

2015 MLG 11

2015 MLG 110

2015_MLG_115

2015 MLG 116

2015 MLG-117

2015_MLG_118

$2015^{-} \mathrm{MLG}^{-} 2$

2015_MLG_3

2015 MLG 4

2015 MLG 50

2015 MLG 51

2015 MLG 52

2015 MLG 53

2015 MLG 54

2015 MLC

2015_MLG_57

2015_MLG_58

2015 MLG 59

2015 MLG_6

2015 MLG 60

2015_MLG_61
2015_MLG_62 2015_MLG_63

2015 MLG 64 2015 MLG 7 2015 MLG-73 2015 MLG 74 2015_MLG_77

2015 MLG 78 2015 MLG 79 2015 MLG 8 2015_MLG_80 2015 MLG 81 2015 MLG 82 2015_MLG_83 2015_MLG_85 2015_MLG_86 2015 MLG 87 2015 MLG 88 2015 MLG 9 2015_MLG_94 2015_MLG_95 2015_MLG_98 O49

Priming294

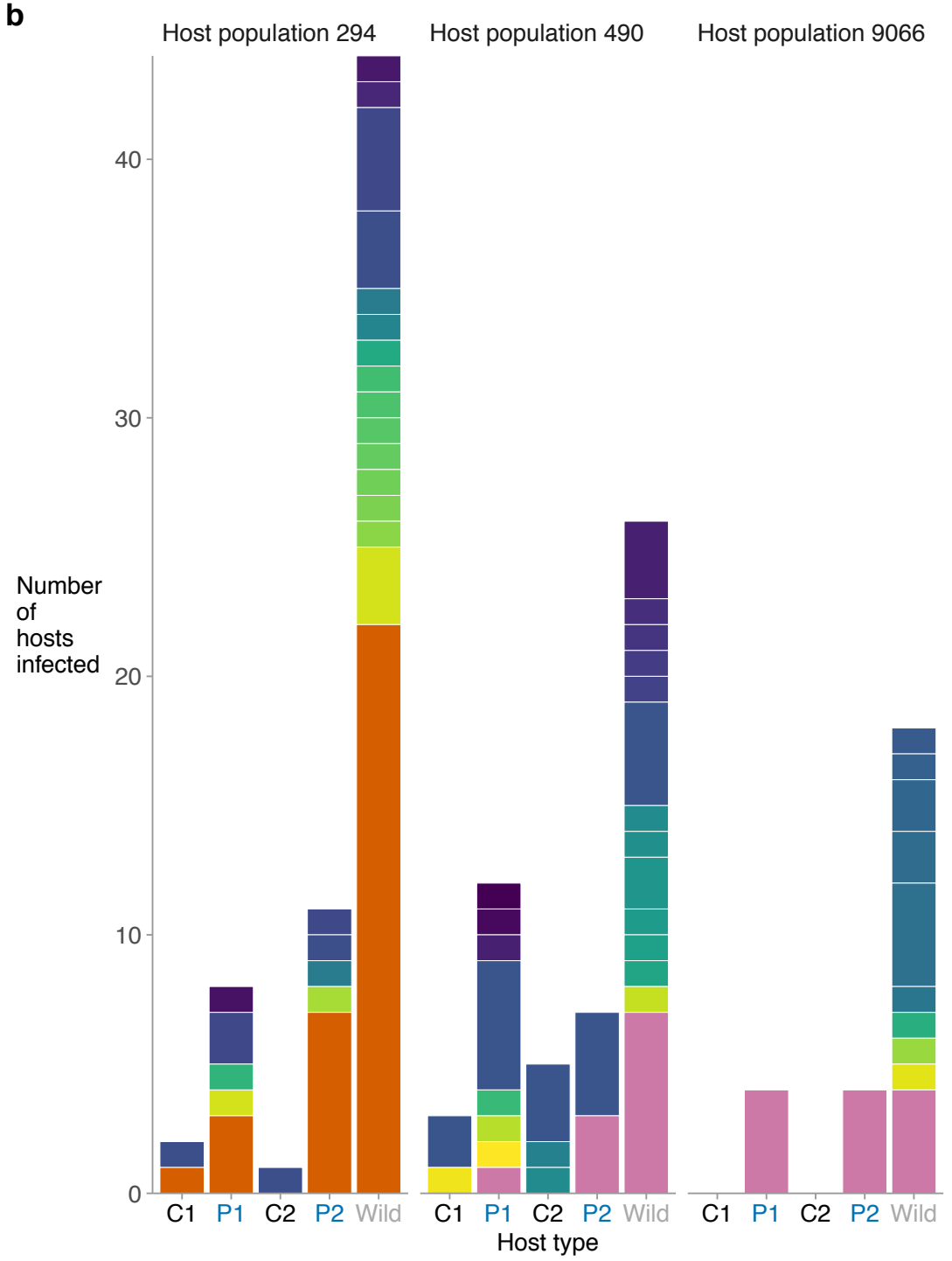

Figure 3 


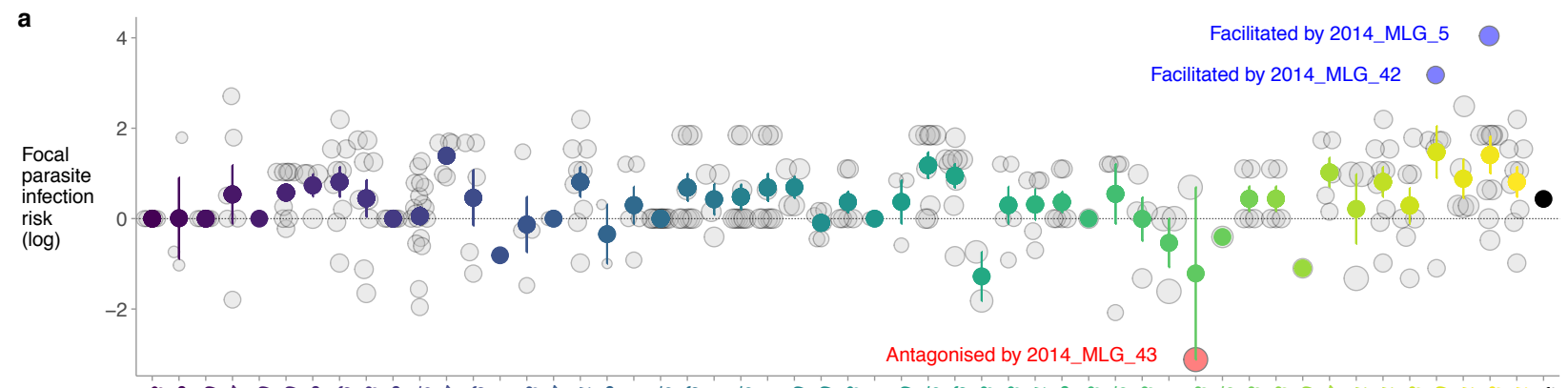

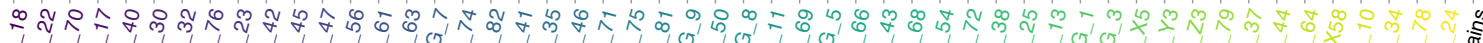

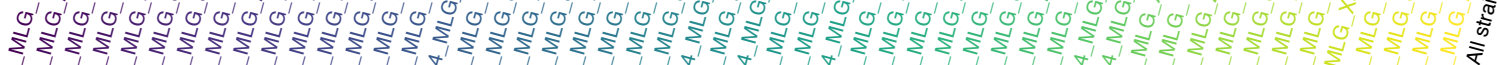

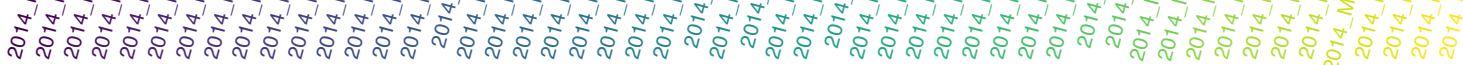

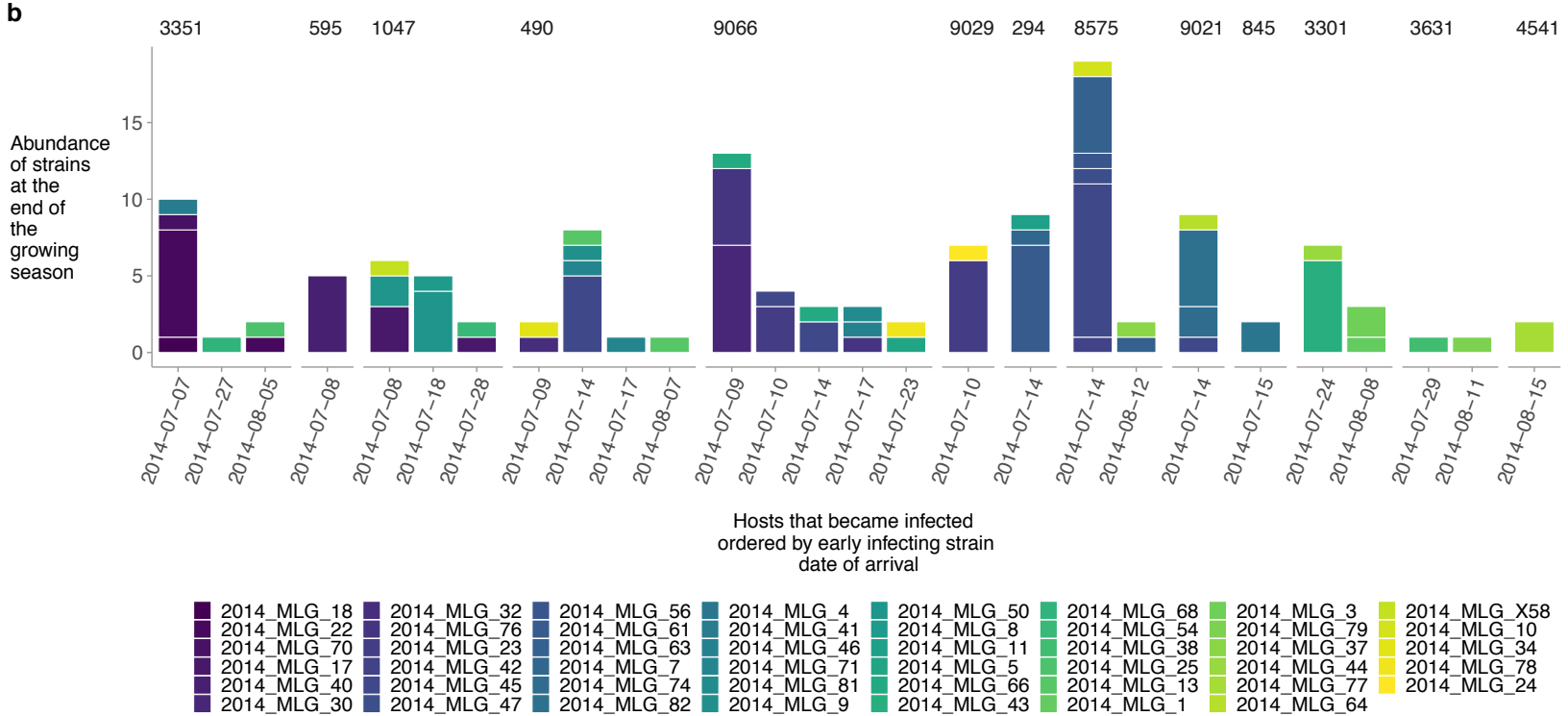

